\title{
Diffusion of chain molecules and mixtures in carbon nanotubes: The effect of host lattice flexibility and theory of diffusion in the Knudsen regime
}

\author{
S. Jakobtorweihen ${ }^{\text {a) }}$ \\ Department of Chemical Reaction Engineering, Hamburg University of Technology, Eissendorfer \\ Strasse 38, D-21073 Hamburg, Germany \\ C. P. Lowe \\ Van 't Hoff Institute for Molecular Sciences (HIMS), University of Amsterdam, Nieuwe Achtergracht 166, \\ 1018 WV Amsterdam, The Netherlands \\ F. J. Keil \\ Department of Chemical Reaction Engineering, Hamburg University of Technology, Eissendorfer \\ Strasse 38, D-21073 Hamburg, Germany \\ B. Smit \\ Van 't Hoff Institute for Molecular Sciences (HIMS), University of Amsterdam, Nieuwe Achtergracht 166, \\ 1018 WV Amsterdam, The Netherlands and Centre Européen de Calcul Atomique Moléculaire \\ (CECAM), Ecole Normale Supérieure, 46 Allée d'Italie, 69364 Lydon, Cedex 7, France
}

(Received 25 April 2007; accepted 5 June 2007; published online 13 July 2007)

A novel algorithm for modeling the influence of the host lattice flexibility in molecular dynamics simulations is extended to chain-like molecules and mixtures. This technique, based on a Lowe-Andersen thermostat, maintains the advantages of both simplicity and efficiency. The same diffusivities and other properties of the flexible framework system are reproduced. Advantageously, the computationally demanding flexible host lattice simulations can be avoided. Using this methodology we study the influence of flexibility on diffusion of $n$-alkanes inside single-walled carbon nanotubes. Furthermore, results are shown for diffusion of two mixtures (methane-helium and ethane-butane). Using these results we investigate the accuracy of theories describing diffusion in the Knudsen regime. For the dynamics in carbon nanotubes the Knudsen diffusivities are much too low. The Smoluchowski model gives better results. Interestingly, the extended Smoluchowski model can reproduce our simulation results obtained with a rigid host lattice. We modify this model to also treat collisions with a flexible interface correctly. As the tangential momentum accommodation coefficient is needed for the theoretical models, we introduce a simple concept to calculate it. (C) 2007 American Institute of Physics.

[DOI: $10.1063 / 1.2753477$ ]

\section{INTRODUCTION}

Nanoporous materials are widely used in industrial applications (e.g., separation processes and catalysis). Because of their importance, it is crucial to gain more insight into the behavior of guest molecules in such porous structures and to find new materials with improved properties. To date, the most widely used nanoporous materials are zeolites. ${ }^{1}$ Currently, a considerable amount of work is in progress investigating the possibility of using carbon nanotubes (CNTs) as host structures for gas separation. In particular, the faster diffusion, compared to zeolites, of guest molecules inside CNTs make them a promising material for membranes. ${ }^{2}$ The first CNT membranes have been produced on a laboratory scale, yielding encouraging results. ${ }^{3-7}$ Economically, it is not yet feasible to produce CNT membranes for industrial large scale applications. However, CNT synthesis is a rapidly developing field and novel synthesis techniques are continually being developed. ${ }^{8-13}$

\footnotetext{
${ }^{a)}$ Author to whom correspondence should be addressed. Electronic mail: jakobtorweihen@tuhh.de
}

To efficiently use CNTs in separation devices it is essential to understand the dynamics of the adsorbates. Molecular simulation is a powerful tool for studying phenomena on a microscopic level. ${ }^{14,15}$ In particular, our understanding of processes inside nanoporous materials can be improved with the aid of these simulations because much of the information obtained from simulations is not accessible experimentally. The promising properties of CNTs motivate molecular dynamics studies of the dynamics of adsorbates within them. ${ }^{16-41}$ Thus far, only a few studies deal with the behavior of alkanes in nanotubes. ${ }^{34-44}$ Further, Wei and Srivastava ${ }^{20}$ investigated the behavior of polymers in CNTs.

Recently we showed that the thermal motion of the carbon atoms making up a CNT plays a crucial role in the dynamics of guest molecules. ${ }^{16}$ This "flexibility" influence was observed for low loadings. For elevated pressures CNT flexibility has no effect. Andreev et al. ${ }^{18}$ showed that this decreases the mobility of water inside nanotubes and influences the hydrophobic behavior of CNTs. In addition, Tuzun et al. $^{33}$ observed an influence of flexibility on the dynamics of guest molecules inside CNTs. Because of the large num- 
ber of carbon atoms involved, and their high degree of connectivity, it is computationally demanding to simulate the nanotube explicitly as a flexible framework. Therefore, we introduced an efficient method to simulate the effect of flexibility on the adsorbates within a simulation using a rigid framework. ${ }^{16,17}$ The technique uses a Lowe-Andersen (LA) thermostat ${ }^{45}$ to mimic the effects of flexibility. This "LoweAndersen interface-fluid collision (LA-IFC) thermostat" is explained in detail in Sec. II A. We used this method to investigate the loading dependent self-diffusion of simple gases ${ }^{16,17}$ and the effects of temperature and CNT size on the diffusion of simple fluids inside CNTs at the zero loading limit. ${ }^{25}$ Chen et al. ${ }^{23}$ used our approach to study the transport diffusion of methane in CNTs and Newsome and Sholl ${ }^{46}$ used it for studying entrance effects. The original LA thermostat $^{45,47}$ was developed for constant temperature (bulk) "dissipative particle dynamics" simulations. Very recently, Koopman and Lowe ${ }^{48}$ showed the advantages of a LA thermostat in constant temperature molecular dynamics simulations. Up until now, the LA-IFC thermostat was parametrized only for simple molecules (one interaction center) and the flexibility influence was investigated only for those kinds of fluids. For practical applications it is important to understand the behavior of more complex molecules (e.g., alkanes) and, of course, mixtures.

The aim of this article is to investigate the influence of CNT flexibility on chain-like molecules and mixtures, and to extend the thermostat methodology to these systems. Subsequently, we investigate the loading dependent self-diffusion of mixtures and pure $n$-alkanes. More specificly, we show results for the zero loading limit for $\mathrm{C} 1-\mathrm{C} 6$, investigate the loading dependence for pure ethane and butane, and show results for two mixtures (methane-helium and ethanebutane). Additionally, we investigated the accuracy of theoretical approaches for diffusion in the Knudsen regime, namely, the Knudsen model, ${ }^{49}$ the Smoluchowski model, ${ }^{50}$ and the extended Smoluchowski model. ${ }^{51}$ We modified the latter so that collisions with a flexible interface are treated correctly. As the "tangential momentum accommodation coefficient" is needed for these models, we have developed a simple concept to calculate this coefficient from standard simulation results.

In the next section the models underlying our simulations are explained, as are details of the simulations. We also explain how the LA-IFC thermostat is extended to mixtures and molecules with more than one interaction center. In Sec. III the effects of flexibility on the diffusion of chain-like molecules are discussed. Results for mixtures are described in Sec. IV. The section thereafter connects the simulation results to theoretical models. Finally, the article is concluded with a discussion.

\section{METHODS}

In this section the methods underlying our simulations are explained. The first subsection describes the thermostat that incorporates the flexibility influence into simulations using rigid frameworks. In particular, the extension to chain molecules and mixtures is introduced.
To distinguish between the different simulation methods we adopt the following conventions. Simulations including a fully flexible carbon nanotube are denoted as "flexible" CNT simulations; simulations with a rigid CNT that take the flexibility into account via the interface-fluid collision thermostat are termed "LA-IFC" simulations while rigid CNT simulations where flexibility is neglected (fluid particles coupled to the Nosé-Hoover chain thermostat) are "rigid CNT" simulations. The size and helicity of carbon nanotubes is specified by two integer numbers $n_{1}$ and $n_{2},{ }^{52}$ so they can be unambiguously labeled as $\left(n_{1}, n_{2}\right)$. All results in this work were obtained with single-walled carbon nanotubes in a zigzag structure $\left(n_{2}=0\right)$.

\section{A. The Lowe-Andersen thermostat for interface-fluid collisions}

Very recently, we introduced an efficient algorithm to avoid expensive flexible CNT simulations. ${ }^{16,17}$ This method yields effectively the same diffusivities and other effects (e.g., energy transfer) as obtained from flexible CNT simulations. It uses a Lowe-Andersen thermostat ${ }^{45}$ which works on interface-fluid collisions. The central idea of this "LoweAndersen interface-fluid collision thermostat" is to mimic the thermal effects of a flexible framework stochastically. To simulate the dynamics of guest molecules inside CNTs this thermostat needs three parameters: the collision radius $r_{\mathrm{LA}}$ and two collision frequencies $\Gamma$. The velocity of a particle is perturbed with a certain probability only if the particle is closer to the CNT than a distance $r_{\mathrm{LA}}$ (collision radius). This defines an interface-fluid collision. Orthogonal to the tube axis ( $x$ and $y$ directions) the guest molecules are restricted by the pore walls and, due to the relatively small pore radius the decorrelation of the velocities in this direction is much faster than for the velocity parallel to the pore axis ( $z$ direction). Although a collision itself is not directionally dependent, we account for the different process orthogonal and parallel to the pore axis by using different thermostat collision frequencies. The orthogonal components of the particle velocity are updated with a probability $\Gamma_{x y} \Delta t$, and the parallel component with a probability $\Gamma_{z} \Delta t$. Here $\Delta t$ is the time step size.

The parallel collision frequency can be calculated from the velocity autocorrelation function (VACF) of the flexible system,

$$
\left\langle v_{z}(0) v_{z}(t)\right\rangle=\left\langle v_{z}(0)^{2}\right\rangle \exp \left[-\frac{\Gamma_{z} \mu}{m_{f}} t\right],
$$

where $v_{z}$ is the velocity in $z$ direction, $\mu=m_{f} m_{C} /\left(m_{f}+m_{C}\right)$ is the reduced mass, $m$ is the mass of a particle, and $t$ is the time. The subscript $f$ indicates a fluid molecule, and the subscript $C$ stands for carbon. A different approach is used to obtain the orthogonal collision frequency. ${ }^{17}$ Within flexible CNT simulations the adsorbed gas is not coupled to a thermostat, so that the thermalizing of the gas (wall-fluid collisions) is a local process. By using local thermalization it is possible to perform a "heating experiment." This involves discontinuously changing the temperature of the flexible CNT or the set temperature in the LA-IFC thermostat (a local thermostat), respectively, and monitoring the time it takes the 
gas to subsequently equilibrate to the new temperature. The orthogonal collision frequency is chosen such that the heating curve $T_{\text {flex }}(t)$ for the flexible system is reproduced as accurately as possible by the Lowe-Andersen thermostat $T_{\text {LA-IFC }}(t)$. This is achieved by minimizing the root-meansquare difference (rmsd) between the two,

$$
\operatorname{rmsd}=\sqrt{\sum_{t}\left[\frac{T_{\mathrm{LA}-\mathrm{IFC}}(t)-T_{\mathrm{flex}}(t)}{T_{\mathrm{flex}}(t)}\right]^{2}} .
$$

Before the thermostat can be used to perform a heating experiment the collision radius must be chosen. $\Gamma_{z}$ calculated from Eq. (1) corresponds to a collision radius equal to the CNT radius $r_{\text {CNT }}$ because all particles are taken into account. To recalculate $\Gamma_{z}$ for different $r_{\mathrm{LA}}$ the probability $P\left(r_{\mathrm{LA}}\right)$, that a molecule is located within a spherical shell of radius $r_{\mathrm{LA}}$, is used,

$$
\Gamma_{z}\left(r_{\mathrm{LA}}\right)=\frac{\Gamma_{z}\left(r_{\mathrm{LA}}=r_{\mathrm{CNT}}\right)}{P\left(r_{\mathrm{LA}}\right)} .
$$

By fitting the parallel collision frequency to a VACF [see Eq. (1)] obtained in a flexible simulation, the collision frequency takes other effects leading to a decorrelation of the velocities into account not just flexibility. These are effects that are present in rigid and flexible CNT simulations. By using such a frequency in rigid simulations, a so-called double counting effect could occur. ${ }^{17}$ One condition for which the influence of the double counting cannot be neglected is a similar decay of the VACFs for the flexible case and the rigid case. ${ }^{17}$ An additional step is necessary to avoid the double counting, in other words to obtain collision frequencies that only take the flexibility effect into account. With the LA-IFC parameters [obtained with Eqs. (1)-(3)] one can perform a molecular dynamics simulation at the zero loading limit. From the VACF calculated in this limit, a collision frequency $\Gamma_{z}^{\text {output }}$ can be calculated [Eq. (1)]. If the effect of double counting is negligible this frequency is equal to the frequency obtained in the corresponding flexible simulation $\Gamma_{\text {flex }}$. If not, a new collision frequency can be calculated,

$$
\Gamma_{\mathrm{LA}-\mathrm{IFC}}^{\text {new-input }}=\left[\Gamma_{\text {flex }}-\left(\Gamma_{\mathrm{LA}-\mathrm{IFC}}^{\text {output }}-\Gamma_{\mathrm{LA}-\mathrm{IFC}}^{\text {input }} P\left(r_{\mathrm{LA}}\right)\right)\right] P\left(r_{\mathrm{LA}}\right)^{-1}
$$

In simulations using the LA-IFC thermostat a frequency is used as an input parameter (superscript input); and from the VACF, obtained in these simulations, a new frequency can be calculated (superscript output). Here, all frequencies are for the $z$ direction (parallel collision frequencies). More details of the double counting effect can be found in Ref. 17 .

The thermostat parameters are only used to determine whether or not a thermostat perturbation of the molecular velocity is necessary. They are not used for the update itself. The equation for the update is a direct result of the original Lowe-Andersen thermostat introduced by Lowe, ${ }^{45}$

$$
v_{f, \alpha}^{\text {new }}=v_{f, \alpha}^{\text {old }}+\frac{\mu}{m_{f}}\left(\xi_{1} \sqrt{\frac{k_{B} T}{m_{C}}}-v_{f, \alpha}^{\text {old }}\right)+\xi_{2} \sqrt{\frac{\mu k_{B} T}{m_{f}^{2}}},
$$

where $\alpha$ represents the direction of the velocity $v, k_{B}$ is the Boltzmann constant, $T$ is the temperature, and $\xi_{1}$ and $\xi_{2}$ are independent random numbers from a Gaussian distribution with zero mean and unit variance.

As yet, we have considered only simple molecules and pure components. The extension to mixtures is very simple. As the thermostat takes only fluid-interface effects into account, all parameters are obtained at the zero loading limit. Parameters obtained for the pure components can be used directly for mixture simulations. The proof for this extension is shown in Sec. IV. Furthermore, we only simulated molecules which can be modeled as one interaction center. For technical applications it is important to study the behavior of more complex molecules. The interaction centers of these molecules have individual velocities that are coupled via the internal interactions (e.g., bond stretch, bond bending, and bond torsion). Because of this coupling, a change of the velocity of one center will influence the velocity of the other centers. Thus, thermalizing one center will have an effect on the entire molecule. To avoid complex algorithms in which the exact collision frequencies for the individual centers are necessary, we determine only one collision frequency for the entire molecule. Therefore, we calculate the parallel collision frequency from the center of mass VACF [see Eq. (1)]. The orthogonal frequency is again fitted to heating curves, as explained above. Note that it is important to recalculate the frequencies [see Eq. (4)], as the flexibility influence is decreasing with chain length. The weaker the flexibility influence the more important the recalculation. ${ }^{17}$

A molecule is a candidate for thermalization only when its center of mass is located closer to the CNT (defined by the positions of the carbon wall atoms) than a distance $r_{\mathrm{LA}}$ (see Fig. 1). Then only one center will be thermalized as this influences the entire molecule. Since the idea was to thermalize on interface-fluid collisions, we update the velocity of the center closest to the CNT. Of course, it would be possible to use the distance of the closest center as the thermalization criterion instead of the center of mass. As this criterion would lead to an increased number of calculations to identify the closest center, we use the center of mass as a computational faster measure (closest center have to be identified only if a velocity is, in fact, recalculated). Moreover, the chosen method produces satisfactory results. The $x$ and $y$ components of the velocity of the center closest to the CNT are updated with a probability $\Gamma_{x y} \Delta t$ and the $z$ component with a probability $\Gamma_{z} \Delta t$. From a physical point of view it is reasonable to update only one center because a collision will always be a local event within the colliding molecule. However, alternative schemes are possible; for instance, a new center of mass velocity could be calculated and in turn the individual velocities could be changed accordingly. As the method we describe here produces satisfactory results we did not investigate alternatives. To check whether or not the thermostat influences the internal structure of a molecule we sampled the internal conformations (e.g., bond length, bond 


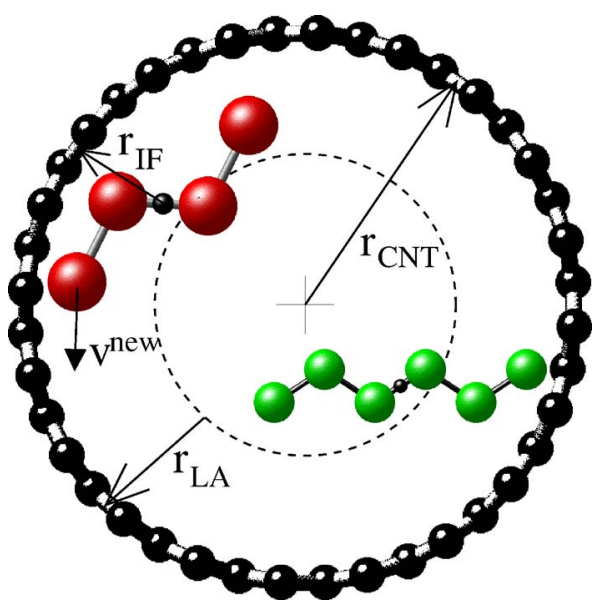

FIG. 1. (Color online) Schematic illustration of the Lowe-Andersen radius (collision radius) $r_{\mathrm{LA}}$, in the $x-y$ plane. The distance of the butane center of mass to the pore wall $r_{\mathrm{IF}}$ is smaller than $r_{\mathrm{LA}}$. Therefore, this molecule will be considered for thermalization. But only the interaction center with the shortest distance to the pore wall will be thermalized (with a certain probability). The $z$ component has a probability to be updated of $\Gamma_{z} \Delta t$, and the $x$ and $y$ components have a probability to be updated of $\Gamma_{x y} \Delta t$. The hexane molecule is nonthermalized, as the center of mass is too far away from the pore wall, although one interaction center of hexane is very close to the pore wall.

bending, and bond torsion). No influence was observed as the different types of simulations (flexible, LA-IFC, and rigid) result in the same structures.

To summarize, the LA-IFC thermostat proceeds as follows (see Fig. 1):

- After each time step calculate the distance $r_{\mathrm{IF}}$ between the interface, and the center of mass of a fluid molecule.

- If $r_{\mathrm{IF}}<r_{\mathrm{LA}}$, determine the nearest center to the interface, the thermostat will act only on this center.

- Update the $z$ component of the velocity of this center with the probability $\Gamma_{z} \Delta t$ and the $x$ and $y$ components with a probability $\Gamma_{x y} \Delta t$; the velocities are updated with Eq. (5).

All LA-IFC parameters used in this work are shown in Table I. The collision radii were chosen according to the maximum of the center of mass radial distribution function.

\section{B. Force fields}

Flexible carbon nanotubes were modeled with the force field of Walther et al. ${ }^{53}$ Where bond vibrations were modeled

TABLE I. Parameter sets for the LA-IFC thermostat $(T=300 \mathrm{~K})$. The $(20,0)$ and the $(13,0)$ CNTs have a radius of 0.78 and $0.51 \mathrm{~nm}$, respectively.

\begin{tabular}{lcccc}
\hline \hline Component & $\mathrm{CNT}$ & $\begin{array}{c}r_{L A} \\
(\mathrm{~nm})\end{array}$ & $\begin{array}{c}\Gamma_{x y} \\
\left(10^{11} \mathrm{~s}^{-1}\right)\end{array}$ & $\begin{array}{c}\Gamma_{z} \\
\left(10^{10} \mathrm{~s}^{-1}\right)\end{array}$ \\
\hline Helium & $(20,0)$ & 0.30 & 6.5 & 3.8 \\
SF $_{6}$ & $(20,0)$ & 0.44 & 1.3 & 2.3 \\
Methane & $(20,0)$ & 0.36 & 3.5 & 5.0 \\
Ethane & $(20,0)$ & 0.38 & 2.6 & 4.9 \\
Propane & $(20,0)$ & 0.39 & 5.6 & 6.0 \\
Butane & $(20,0)$ & 0.40 & 6.1 & 7.2 \\
Pentane & $(20,0)$ & 0.41 & 6.4 & 7.3 \\
Hexane & $(20,0)$ & 0.41 & 8.1 & 9.1 \\
Butane & $(13,0)$ & 0.51 & 3.7 & 2.3 \\
\hline \hline
\end{tabular}

TABLE II. Lennard-Jones parameters and their source.

\begin{tabular}{lccc}
\hline \hline Center & $\sigma(\AA)$ & $\epsilon / k_{B}(\mathrm{~K})$ & Ref. \\
\hline $\mathrm{CH}_{4}$ & 3.73 & 148 & 54 \\
$\mathrm{CH}_{3}$ & 3.75 & 98 & 54 \\
$\mathrm{CH}_{2}$ & 3.95 & 46 & 54 \\
$\mathrm{Helium}_{\mathrm{SF}_{6}}$ & 2.551 & 10.22 & 64 \\
Carbon & 5.128 & 222.1 & 64 \\
\hline \hline
\end{tabular}

with a Morse potential, bond bending was controlled by a harmonic cosine potential, and bond torsions with a two-fold torsion potential. The following small modifications were employed. Equilibrium bond lengths and equilibrium bend angles were set to the values of the rigid CNT. We have not used intermolecular carbon-carbon interactions. Comparisons with results where these interactions were taken into account showed that they have no influence.

Intermolecular interactions were modeled with the shifted and truncated Lennard-Jones 12-6 potential, for which a cut off radius of $14 \AA$ was used. The fluid molecules were modeled as united atoms. For the alkanes the TraPPE force field was used, ${ }^{54}$ where the bond angle bending is described by a harmonic potential and the bond torsion with a three-cosine-fourier potential. In the original TraPPE force field the bond lengths are fixed. In order to avoid constrained dynamics, we used a harmonic potential for the bond vibration. ${ }^{55}$ The carbon Lennard-Jones parameters, which are widely accepted for carbon, ${ }^{21}$ were taken from Ref. 56. Lorentz-Berthelot mixing rules were used to determine the interactions of unlike molecular centers. The Lennard-Jones parameters are listed in Table II.

\section{Computational details}

We performed molecular dynamics simulations using the velocity Verlet integration scheme ${ }^{14}$ in combination with the reversible reference system propagation algorithm, ${ }^{14,57}$ where a short time step of $0.5 \mathrm{fs}$ and a long time step of $2 \mathrm{fs}$ were used. The system was equilibrated by following the particle dynamics for $1 \mathrm{~ns}$. In the sampling phase of simulations using a rigid CNT the particle dynamics were followed for 400-1000 ns (depending on the system). These simulations allowed the use of interpolation grids for the carbonfluid interactions. This technique saves a considerable amount of CPU time. Unfortunately it cannot be used for flexible CNT simulations, where the particle dynamics were followed for 120-400 ns. All pure component simulations were performed with 64 molecules. For the zero loading limit simulations these are noninteracting molecules (no intermolecular fluid-fluid forces). The CNT length was adjusted to simulate the system at the correct loading, and for zero loading it was at least $5.1 \mathrm{~nm}$. In mixture simulations we used a system size such that 64 molecules of the lowest concentrated component were present. To obtain which composition and loading corresponds to a given pressure, we performed Monte Carlo simulations in the grand-canonical ensemble. In this work we did not investigate temperature effects, and all systems were studied at the same temperature 


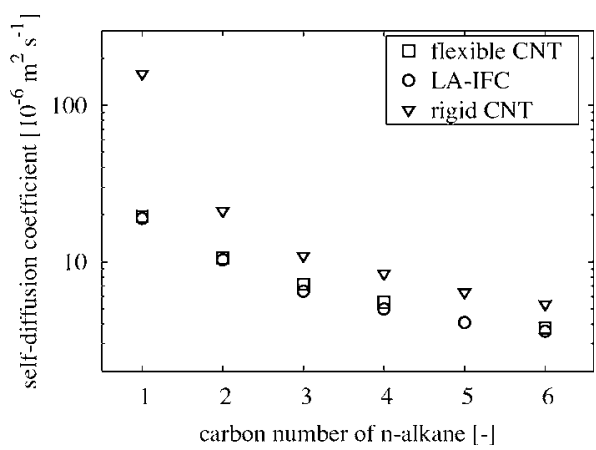

FIG. 2. Self-diffusion coefficients of $n$-alkanes inside a $(20,0)$ CNT for the zero loading limit at $300 \mathrm{~K}$, calculated with a flexible CNT, with a rigid nanotube without taking the flexibility effect into account, and with the LA-IFC thermostat to take the flexibility influence into account.

of $300 \mathrm{~K}$. To keep it constant in simulations where the flexible effect is not taken into account the fluid molecules were coupled to the Nosé-Hoover chain thermostat. ${ }^{58}$ In simulations using a flexible CNT the carbon atoms of the tube were coupled to the Nosé-Hoover chain thermostat and the fluid temperature was kept constant by the flexible framework. All self-diffusion coefficients $D_{S}$ were calculated only for motion parallel to the nanotube axis (one-dimensional) using the Einstein definition

$$
D_{S}=\frac{1}{2} \lim _{t \rightarrow \infty} \frac{d}{d t}\left\langle[z(t)-z(0)]^{2}\right\rangle .
$$

Initially a nondiffusive regime is observed, the mean-squared displacement (MSD) $[z(t)-z(0)]^{2}$ is not linear in time. The linear fit to the MSD is applied only for the linear regime. All results shown in this work are averaged over five independent simulations. In the figures the error bars are not shown when they are smaller than the symbol size.

\section{THE EFFECT OF FLEXIBILITY ON DIFFUSION OF CHAIN MOLECULES}

Recently, we have shown that for diffusion at low loadings CNT flexibility can be very important. ${ }^{16,17}$ The most pronounced influence is observed in the zero loading limit. In our previous studies we investigated the influence on simple gases. In Fig. 2 results for $n$-alkanes for the zero loading limit are shown. For comparison all results were obtained in a $(20,0)$ single-walled carbon nanotube $\left(r_{\mathrm{CNT}}\right.$ $=0.78 \mathrm{~nm}$ ). As expected the self-diffusivities decrease monotonically with the alkane length. CNTs are straight channels with a constant size over the channel length. In contrast, for cage-type zeolites the chain-length dependent diffusion can be nonmonotonic. ${ }^{59}$ As it corresponds to the monotonic behavior of self-diffusivities, we note that the logarithms of the Henry constants and the heats of adsorption also show a monotonic chain-length dependence in CNTs. ${ }^{42}$ The influence of tube flexibility on the diffusion coefficients is also decreasing with the alkane length. Although it is already small for hexane, it appears that it never completely vanishes with chain length. The decreasing influence is because the chain-like molecules have a higher probability of colliding with the wall. It is obvious that the higher the number of interaction centers the higher the probability that at least one
TABLE III. Self-diffusion coefficients for the zero loading limit at $300 \mathrm{~K}$. Errors are given in the subscripts. The $(20,0)$ and the $(13,0)$ CNTs have a radius of 0.78 and $0.51 \mathrm{~nm}$, respectively. Note that the results for methane are taken from Ref. 17.

\begin{tabular}{|c|c|c|c|}
\hline Component & CNT & Simulation method & $D_{S}\left(10^{-6} \mathrm{~m}^{2} \mathrm{~s}^{-1}\right)$ \\
\hline \multirow[t]{3}{*}{ Methane } & \multirow[t]{3}{*}{$(20,0)$} & Flexible & $19.42_{0.42}$ \\
\hline & & LA-IFC & $18.92_{0.31}$ \\
\hline & & Rigid & $159.09_{3.00}$ \\
\hline \multirow[t]{3}{*}{ Ethane } & \multirow[t]{3}{*}{$(20,0)$} & Flexible & $10.59_{0.27}$ \\
\hline & & LA-IFC & $10.30_{0.21}$ \\
\hline & & Rigid & $21.17_{0.35}$ \\
\hline \multirow[t]{3}{*}{ Propane } & \multirow[t]{3}{*}{$(20,0)$} & Flexible & $7.18_{0.31}$ \\
\hline & & LA-IFC & $6.49_{0.06}$ \\
\hline & & Rigid & $10.94_{0.22}$ \\
\hline \multirow[t]{3}{*}{ Butane } & \multirow[t]{3}{*}{$(20,0)$} & Flexible & $5.52_{0.21}$ \\
\hline & & LA-IFC & $5.00_{0.08}$ \\
\hline & & Rigid & $8.42_{0.27}$ \\
\hline \multirow[t]{2}{*}{ Pentane } & \multirow[t]{2}{*}{$(20,0)$} & LA-IFC & $4.10_{0.10}$ \\
\hline & & Rigid & $6.39_{0.20}$ \\
\hline \multirow[t]{3}{*}{ Hexane } & \multirow[t]{3}{*}{$(20,0)$} & Flexible & $3.79_{0.36}$ \\
\hline & & LA-IFC & $3.60_{0.03}$ \\
\hline & & Rigid & $5.38_{0.05}$ \\
\hline \multirow[t]{3}{*}{ Butane } & \multirow[t]{3}{*}{$(13,0)$} & Flexible & $6.09_{0.12}$ \\
\hline & & LA-IFC & $5.31_{0.14}$ \\
\hline & & Rigid & $8.38_{0.15}$ \\
\hline \multirow[t]{3}{*}{$\mathrm{SF}_{6}$} & \multirow[t]{3}{*}{$(20,0)$} & Flexible & $21.62_{1.17}$ \\
\hline & & LA-IFC & $21.96_{0.39}$ \\
\hline & & Rigid & non-diffusive \\
\hline
\end{tabular}

center is close to the pore wall and undergoes a collision. Due to the coupling via the bonds, every collision of a single interaction center affects the entire molecule and changes the center of mass velocity. In other words, the molecules modeled with multiple centers have a "roughness" themselves. That only an increase in the number of interaction centers leads to a reduced flexibility influence and not an increased size of a single interaction center is evident by comparing the results for methane and sulfur hexafluoride in Table III. Both are modeled as a single interaction center. For the larger $\mathrm{SF}_{6}$, the flexibility influence is not reduced, rather, it is enhanced (see also Fig. 6 of Ref. 17). A discussion on the influence of the size parameter can be found in Ref. 17.

To investigate the influence of the tube diameter, we compared the self-diffusivities for butane inside the $(20,0)$ tube with diffusivities of butane inside a $(13,0)$ CNT $\left(r_{\mathrm{CNT}}\right.$ $=0.51 \mathrm{~nm}$ ). The data in Table III show that in the smaller nanotube the flexibility influence is also present. Chen et $a l .{ }^{23}$ observed a distinct influence of flexibility on the transport diffusion of simple gases. Thus, we conclude from our results that a flexibility influence on transport diffusion is present also for more complex molecules. The magnitude of the effect is just reduced relative to simple gases.

Figure 2 shows that the LA-IFC thermostat for chain molecules, as proposed in Sec. II, can indeed reproduce the flexible results. All LA-IFC results slightly underpredict the 


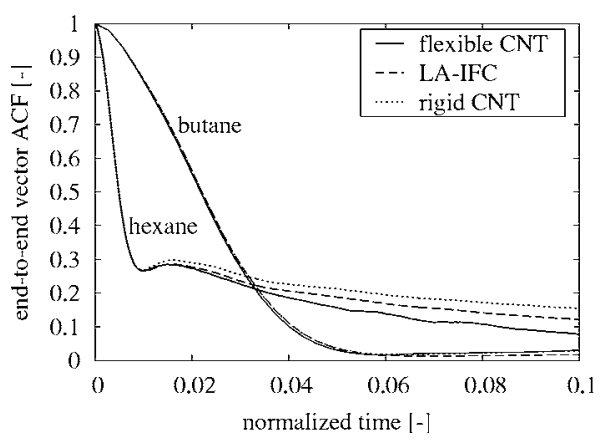

FIG. 3. End-to-end vector autocorrelation functions for $n$-butane and $n$-hexane, calculated with a flexible CNT, with a rigid nanotube without taking the flexibility effect into account, and with the LA-IFC thermostat to take the flexibility influence into account. For a better comparison of the butane and the hexane results we normalized the time. For the three butane results we used the time corresponding to a vanishing ACF of the LA-IFC butane result. For the three hexane results we used the time corresponding to a vanishing ACF of the LA-IFC hexane result.

flexible results. This is because after the recalculation of the collision frequencies we still observe a small double counting effect. All absolute deviations (flexible versus LA-IFC) are between $(0.2$ and 0.7$) \times 10^{-6} \mathrm{~m}^{2} \mathrm{~s}^{-1}$ (see Table III). A correlation between the chain length and absolute deviation is not observed. To check whether the results are robust with respect to the choice of collision radius, for some systems different sets of LA-IFC parameters were calculated. These results showed no remarkable deviations compared to the results described in this work. Hence, it is evident that with a small extension of the new thermostat the effect of flexibility on diffusion of more complex molecules is reproduced. Furthermore, as for simple molecules, ${ }^{17}$ it gives the same heat transfer across the rigid interface as across a flexible interface. Note that the extended methodology keeps its simplicity.

As mentioned, the thermalization does not influence the internal structure of the molecules. To investigate the influence on the rotational motion we calculated the end-to-end vector autocorrelation function. This is the scalar product of the normalized end-to-end vector at a time $t_{0}$ and the normalized end-to-end vector at a time $t$ later. Therefore, it is a measure of the rate of orientational relaxation. Figure 3 shows that flexibility has nearly no influence on the rotational motion for butane, and the new thermostat reproduces this. In contrast, the hexane rotational motion is slightly influenced by flexibility. The results obtained with the LA-IFC thermostat are closer to the more realistic flexible result, although the agreement is moderate. Note that we did not include the rotational motion explicitly in the parameter calculation so the results shown in Fig. 3 illustrate another advantage of our method. Note that the Lowe-Andersen thermostat can be implemented such that the angular momentum is conserved. $^{45,48}$

One of the advantages of the LA-IFC thermostat is that once the parameters are obtained they can be used for any loading of that system. ${ }^{16,17}$ In Fig. 4 we show the loadingdependent self-diffusion coefficients for ethane and butane. The variation of the coefficients over pressure is two orders of magnitude. For all pressures the diffusion of the smaller

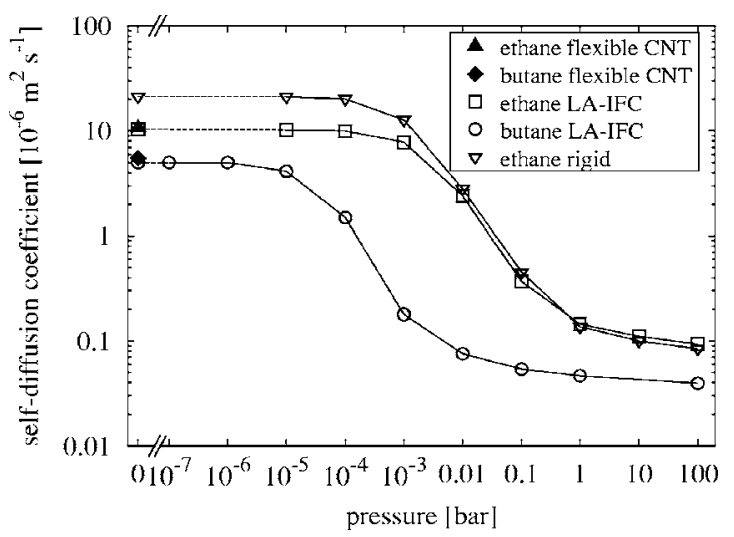

FIG. 4. Self-diffusion coefficients of pure ethane and pure butane inside a $(20,0) \mathrm{CNT}$ at $300 \mathrm{~K}$, obtained with the LA-IFC thermostat to take the flexibility influence into account, compared to results obtained with a flexible CNT. The lines are added to guide the eye.

ethane molecule is faster. Both adsorbates show a constant self-diffusion for low loadings, because the fluid molecules hardly ever collide so that the whole process is determined by fluid-wall collisions. By increasing the pressure/loading the diffusivities decrease and as the loading approaches saturation the slope becomes very small. As expected, the diffusivities of the stronger adsorbing butane starts to decrease at a lower pressure than the ethane self-diffusion coefficients. In addition, for butane the saturation loading is reached at lower pressures. By comparing loading-dependent diffusivities of ethane obtained with the LA-IFC thermostat with results where the flexibility influence is not taken into account, two features become clear. First, flexibility influences the dynamics at low loadings only, as found earlier for simple molecules. ${ }^{16}$ Second, the flexibility influence at low loadings is not as pronounced as for methane. For ethane the low loading plateau for both rigid and flexible results sets in at a similar pressure. In both cases this is different for methane. ${ }^{16}$

Krishna and van Baten ${ }^{34}$ also obtained loadingdependent self-diffusion coefficients for pure ethane and pure butane inside a $(20,0) \mathrm{CNT}$ at $300 \mathrm{~K}$. They simulated only loadings for which CNT flexibility has no influence. Our results at these conditions are in agreement with their results.

\section{DIFFUSION OF MIXTURES IN SINGLE-WALLED CARBON NANOTUBES}

Here we investigate the behavior of two mixtures with a constant bulk composition. We have chosen the bulk composition such that both species are present with considerable concentration inside the CNT. The adsorbate compositions inside the CNTs and the relations between pressure and loading were calculated from grand-canonical Monte Carlo simulations. $^{14}$

The first mixture we study is a methane-helium mixture with a bulk composition of $y_{\mathrm{CH}_{4}}=0.05$ and $y_{\mathrm{He}}=0.95$. Resulting compositions inside the CNT are shown in the inset of Fig. 5. We chose this mixture because the two species exhibit a markedly different flexibility influence ${ }^{17}$ and they adsorb differently. Figure 5 provides evidence for the correct reproduction of the flexibility influence when the LA-IFC thermo- 


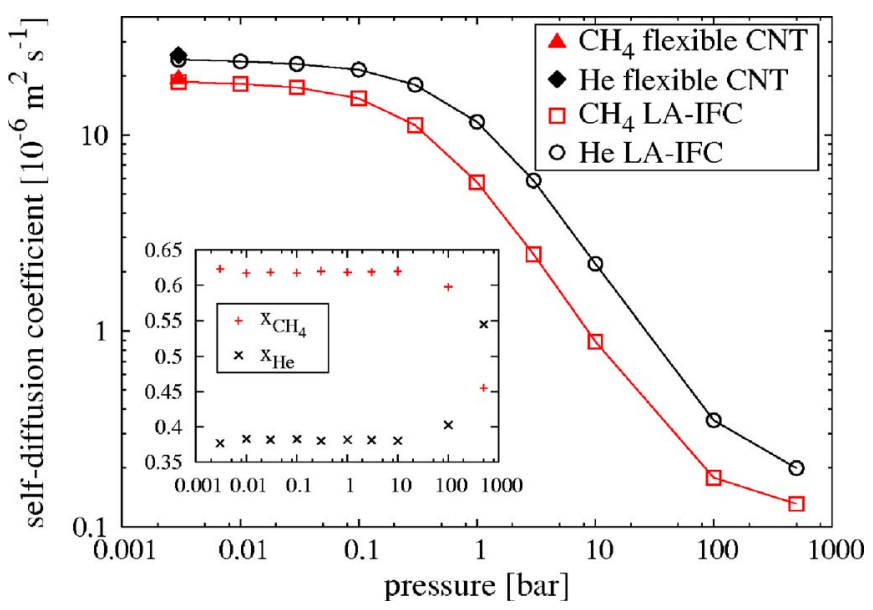

FIG. 5. (Color online) Self-diffusion coefficients of a methane-helium mixture inside a $(20,0) \mathrm{CNT}$ at $300 \mathrm{~K}$, obtained with a rigid nanotube where the flexibility influence is modeled with the LA-IFC thermostat, compared to results obtained with a flexible CNT. The corresponding bulk composition is constant $\left(y_{\mathrm{CH}_{4}}=0.05\right.$ and $\left.y_{\mathrm{He}}=0.95\right)$. The inset shows the mole fractions inside the nanotube as a function of pressure. Lines are added to guide the eye.

stat is used. Thus, the thermostat parameters obtained for the pure components can be used for mixtures, as suggested in Sec. II. For the entire pressure range the diffusion coefficients for the smaller and lighter helium are lower compared with those of methane. The diffusivities at low pressures are in the same range as the diffusivities for the pure components at these conditions (see Table III and Ref. 17). This is to be expected because at very low loadings the molecules hardly collide, so that they do not influence one another. The change in composition starting between 10 and 100 bars does not seem to influence the loading dependence of the selfdiffusion.

The second mixture studied consists of two components, each with more than one interaction center: ethane-butane with a constant bulk concentration of $y_{\mathrm{C} 2}=0.99$ and $y_{\mathrm{C} 4}$ $=0.01$. Resulting compositions inside the CNT are shown in the inset of Fig. 6. For low pressures the pore phase composition is almost constant. With increasing pressure the butane concentration starts to increase, so that it is slightly higher than the ethane concentration. At still higher loadings the smaller ethane is again favorably adsorbed. Due to the constant bulk concentration, this behavior is identical to two changes in the selectivity between 0.05 and 0.5 bar. Both components are technically relevant and both extensions to the LA-IFC thermostat that are introduced in this article have to be applied to this system. Again the influence of the CNT flexibility is reproduced correctly by the thermostat (see Fig. $6)$. For the reasons given above the low loading diffusivities (maximum self-diffusion coefficients) compare well (within the statistical fluctuations) to the pure component diffusivities (see Table III). For the highest loadings investigated ethane diffusion coefficients are smaller than obtained for pure ethane, because the ethane in the mixture diffuses in a system with a considerable amount of slower and larger butane molecules present, which are slowing them down. Under these conditions the diffusivities for ethane and butane are very similar, this is in agreement with the results in

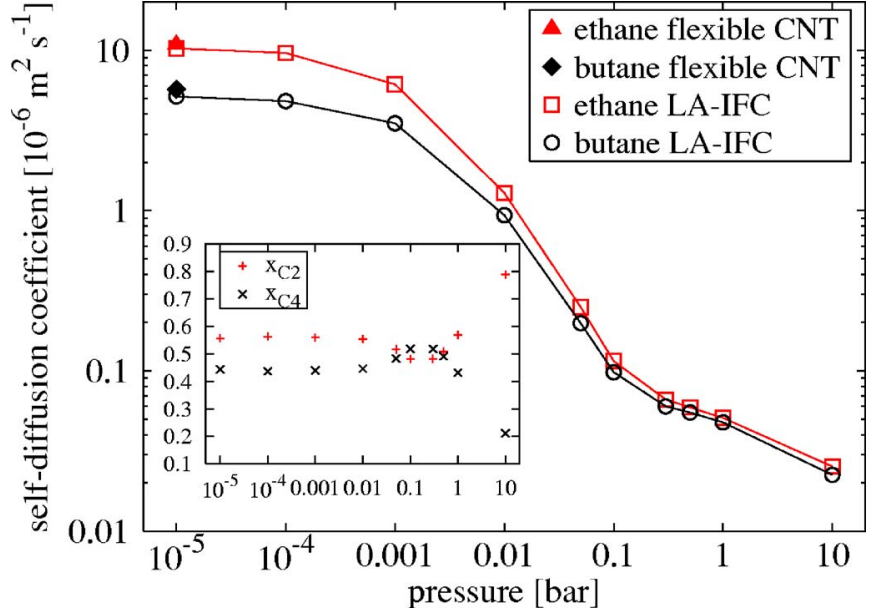

FIG. 6. (Color online) Self-diffusion coefficients of a ethane-butane mixture inside a $(20,0) \mathrm{CNT}$ at $300 \mathrm{~K}$, obtained with a rigid nanotube where the flexibility influence is modeled with the LA-IFC thermostat, compared to results obtained with a flexible CNT. The corresponding bulk composition is constant $\left(y_{\mathrm{C} 2}=0.99\right.$ and $\left.y_{\mathrm{C} 4}=0.01\right)$. The inset shows the mole fractions inside the nanotube as a function of pressure. Lines are added to guide the eye.

Ref. 34. The authors observed strong correlation effects. As a result the self-diffusivities in mixtures are similar. The selectivity changes have only a slight influence on the loadingdependent self-diffusion, which is observable in slope changes. Selectivity changes possibly have more influence on transport diffusivities. However, transport diffusion is beyond the scope of this article, although transport diffusivities could be calculated from molecular simulations.

\section{THEORY OF DIFFUSION IN THE KNUDSEN REGIME}

The regime where the diffusion process is dominated by fluid-interface collisions (large Knudsen number) is called the "Knudsen regime" or "rarefied gas state." As the carbon nanotube flexibility influence is present in this regime and our new thermostat yields correct results also in this limit, here we relate our simulation results to theoretical results. Note that in the zero loading limit the self- and transport diffusivities become equal. The dynamics are completely described by the self-diffusivities. In a recent publication we showed that the Stokes-Einstein equation can easily be related to simulation results in the zero loading limit. ${ }^{25}$ As long ago as 1909 Knudsen introduced a theory of diffusion for the rarefied gas state. ${ }^{49} \mathrm{He}$ introduced the Knudsen diffusivity for diffusion in cylindrical pores with radius $r$ by assuming that every gas-pore collision is diffuse,

$$
D_{S}^{\mathrm{Kn}}=r \sqrt{\frac{32 k_{B} T}{9 \pi m_{f}}} .
$$

Because of the assumption of purely diffuse collisions (velocity after collision is entirely independent of velocity before collision) this equation gives much too low diffusion coefficients. ${ }^{51}$ For the systems studied in this work $D_{S}^{\mathrm{Kn}}$ is up to two orders of magnitude too low. The Smoluchowski model $^{50}$ overcomes the drawback of assuming every collision as diffuse. Rather it is assumed that a single collision is either purely diffuse or purely specular. A certain fraction $f$ of collisions is purely diffuse and a fraction $(1-f)$ is then 
purely specular. The "tangential momentum accommodation coefficient" (TMAC) $f$ represents the fraction of the total collisions which are diffusive,

$$
f=\frac{\text { diffuse collisions }}{\text { diffuse collisions }+ \text { specular collisions }} .
$$

Smoluchowski obtained a model which is dependent on this coefficient,

$$
D_{S}^{\mathrm{Smol}}=D_{S}^{\mathrm{Kn}}\left(\frac{2-f}{f}\right)
$$

Arya $e t a l{ }^{51}$ noted that, especially for weakly adsorbing fluids, the angle of reflection after a collision is dependent on the incident angle and its point of impact at the surface. Consequently each collision is essentially a combination of a specular and a diffuse collision. To account for this they introduced the extended Smoluchowski model,

$$
D_{S}^{\mathrm{extS}}=D_{S}^{\mathrm{Kn}}\left[1+\frac{3 \pi d_{\mathrm{eff}}}{8 r}\left(\frac{1-f}{f}\right)\right],
$$

where $d_{\text {eff }}$ is the effective distance, the average distance a particle travels between two successive collisions. In the latter two models the self-diffusion estimate reduces to the calculation of the TMAC. Arya et al. ${ }^{60}$ determined this coefficient in collision scattering simulations. In these simulations a drift velocity is superimposed on the particles. The results are independent of the chosen drift velocity only at small drift velocities. For hard spheres in rigid slit pores it was shown that the extended Smoluchowski model can reproduce simulation results quantitatively. ${ }^{51}$ The original Smoluchowski model matches the behavior only qualitatively and overpredicts the self-diffusion coefficients. Both theories converge to the Knudsen model for infinitely rough walls $(f \rightarrow 1)$, where every collision is diffuse.

To relate our simulation results to these theories, one has to realize that the collision frequency, obtained from short flexible CNT simulations (see Sec. II), is the number of diffuse collisions per unit time. Hence, to calculate the TMAC we are left with determining the total number of collisions. Molecules inside a nanotube are restricted in the $x-y$ plane. Therefore, the mean-squared displacements for these directions become constant after very short times. To calculate the effective distance/diameter, the average distance between two collisions, we have to use the plateau value of the average absolute displacement $\langle|x(0)-x(t)|\rangle$. Thus, we can calculate the total number of collisions (diffuse and specular),

$$
\Gamma_{\mathrm{all}}=\frac{2\left\langle v_{x}\right\rangle}{\Delta x_{\mathrm{eq}}}=\frac{2}{d_{\mathrm{eff}}} \sqrt{\frac{k_{B} T}{2 \pi m_{f}}},
$$

where $\Delta x_{\text {eq }}$ represents the plateau values of the \langle|$x(0)$ $-x(t)|\rangle$ in the $x$ direction. Here we used the symmetry of CNTs $\left(\Delta x_{\text {eq }}=\Delta y_{\text {eq }}\right.$ and $\left.\left\langle v_{x}\right\rangle=\left\langle v_{y}\right\rangle\right)$. Note that, for chain-like molecules, $\Delta x_{\text {eq }}$ must be sampled for an end bead. The TMAC is now defined as $f=\Gamma_{z, \text { flex }} / \Gamma_{\text {all }}$ and the reciprocal of $\Gamma_{\text {all }}$ is the average collision time $\tau$, that is, the time between two successive collisions. This time corresponds to characteristic points of the VACFs in the $x$ and $y$ directions (see Fig. 7). For methane in a $(20,0)$ and a $(50,0) \mathrm{CNT}$ the VACFs

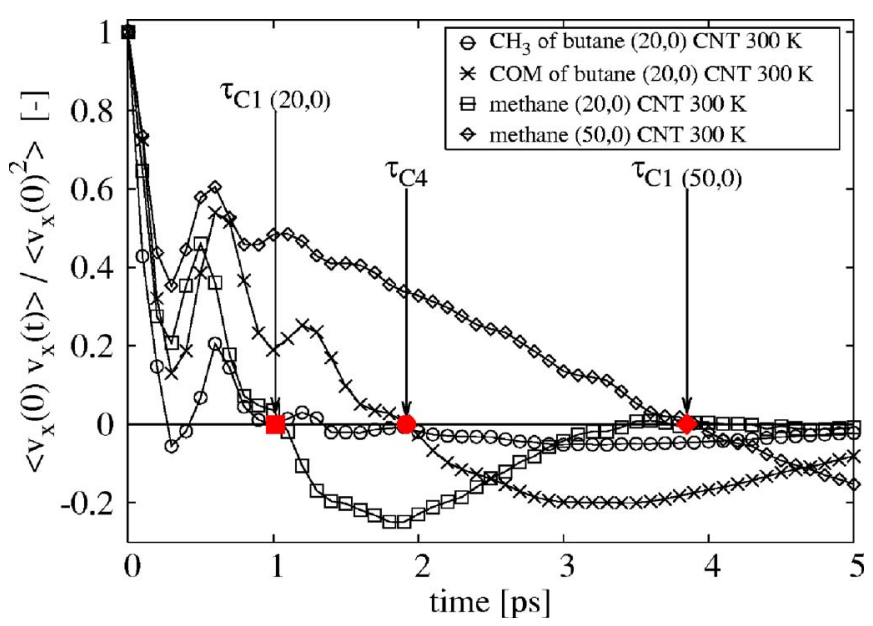

FIG. 7. (Color online) Normalized velocity autocorrelation functions of the $x$ direction for different systems. For butane the VACFs for a $\mathrm{CH}_{3}$ center and for the center of mass (c.m.) are shown. The arrows and the filled symbols mark the collision times $\tau=\Gamma_{\text {all }}^{-1}$ for the different systems. Lines are added to guide the eye.

are equal to zero for the first time. Similarly, for the center of mass of butane in a $(20,0)$ CNT the VACF is also equal to zero for the first time at the collision time, and the VACF of a $\mathrm{CH}_{3}$ center of butane is also zero here (although not for the first time). We note that the values obtained from simulations to calculate $f\left(\Gamma_{z, \text { flex }}\right.$ and $\left.\Delta x_{\mathrm{eq}}\right)$ can be obtained in very short simulations since they represent short time behavior.

Figure 8(a) shows that the original Smoluchowski model predicts the correct diffusivity for propane and overpredicts the diffusivities for smaller molecules and underpredicts it for larger molecules. On the other hand, the extended Smoluchowski model shows a systematic underprediction. In a recent publication we investigated the CNT size influence on diffusion. ${ }^{25}$ Here we use these results to investigate the reliability of the theoretical models. Figure 8(b) shows results for methane at $300 \mathrm{~K}$. Clearly the Smoluchowski model overpredicts the self-diffusion coefficients for small radii and gives a good reproduction for large radii. The extended Smoluchowski model again shows a systematic underprediction. Interestingly, the same relations are observed for all diffusion coefficients shown in Ref. 25 (methane at 300, 500, and $700 \mathrm{~K}$ and helium at 300 and $100 \mathrm{~K}$ ). The relative deviation of the extended Smoluchowski model depends on the species. For all methane results $D_{S}^{\mathrm{th}} / D_{S}^{\mathrm{sim}} \approx 0.43$, and for all helium results $D_{S}^{\text {th }} / D_{S}^{\text {sim }} \approx 0.75$. The deviations of the two butane results obtained in this work are also equal. Simulating model fluids shows that only the mass of the fluid is influencing this deviation, and the Lennard-Jones parameters have no influence. Another property that influences the deviation is the mass of the carbon atoms of the CNT. This is a remarkable observation and leads to the conclusion that the masses are not correctly represented in the extended Smoluchowski model. Arya et al. ${ }^{51}$ derived this model for collisions with rigid interfaces, so the masses of the interface atoms are ignored. In fact, by using simulation results obtained from simulations where the flexibility influence is neglected, the extended Smoluchowski model can indeed quantitatively reproduce the diffusion coefficients for the rigid 

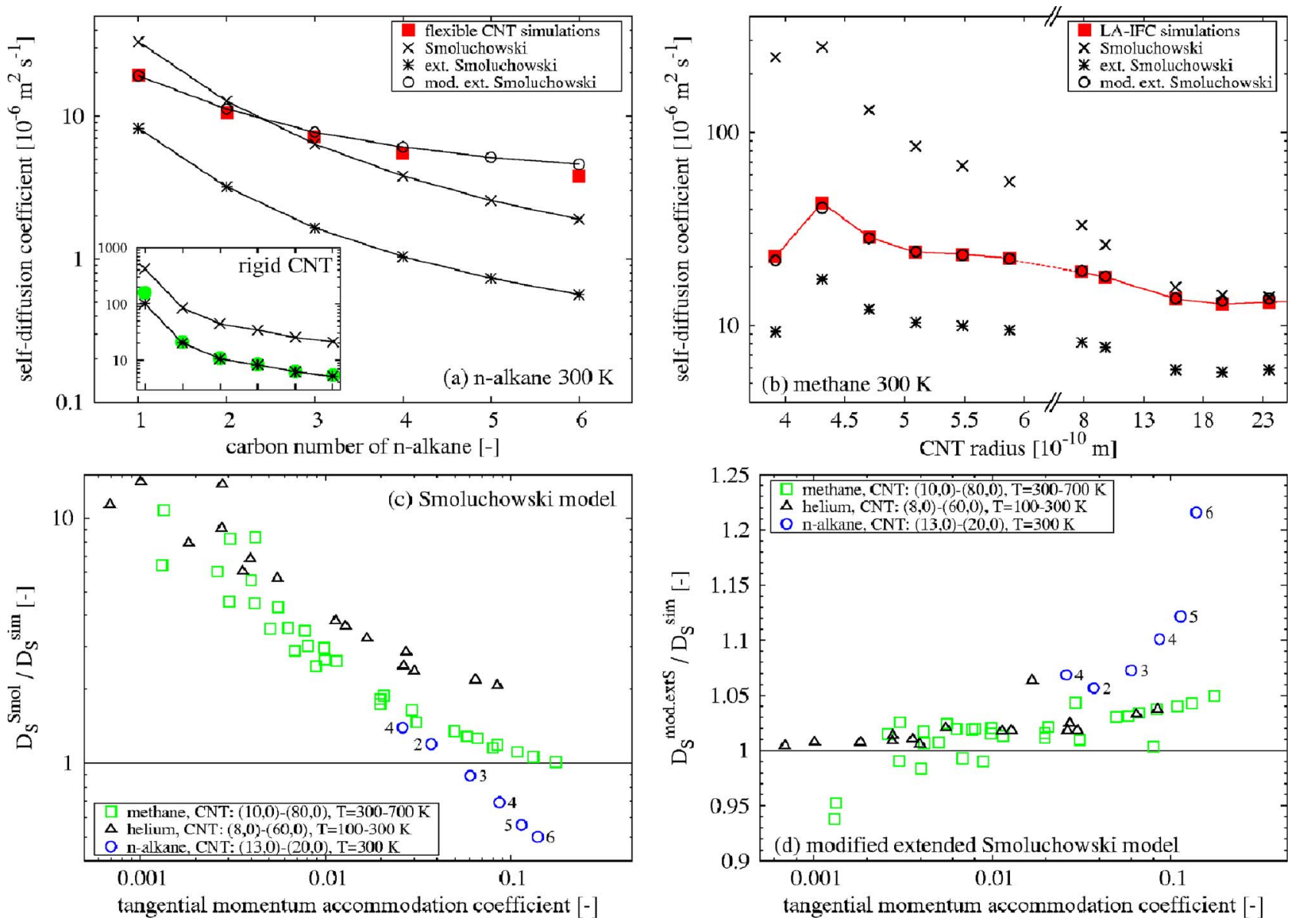

FIG. 8. (Color online) Comparison of self-diffusion coefficients at the zero loading limit obtained in molecular dynamics simulations with different theoretical approaches. If not otherwise mentioned the flexibility influence is taken into account. The simulation results for methane and helium are taken from Ref. 25. The numbers next to the symbols are the carbon number of the $n$-alkane. (a) $n$-alkanes inside a $(20,0) \mathrm{CNT}$ at $300 \mathrm{~K}$. The inset shows the results for the rigid case. (b) Methane at $300 \mathrm{~K}$. Note that the abscissa is not uniform over the whole range. (c) Deviation between the original Smoluchowski model and simulation results. (d) Deviation between the modified extended Smoluchowski model and simulation results. Note that the relative deviations for the extended Smoluchowski model (nonmodified) is constant for each species, for all methane results $D_{S}^{\text {exts }} / D_{S}^{\text {sim }} \approx 0.43$ and for all helium results $D_{S}^{\text {extS }} / D_{S}^{\text {sim }} \approx 0.75$ (not shown here).

case [see inset of Fig. 8(a)]. Here we note that Arya et al. ${ }^{60}$ investigated the influence of wall flexibility on the TMAC only. Since they simulated a light gas confined by very rough walls the flexibility does not enhance the diffuse character of the collisions and $f$ stays unchanged. To obtain correct results for the flexible case $D_{S}^{\text {extS }}$ must be multiplied with a term that accounts for collisions with a flexible interface,

$$
D_{S}^{\text {mod.extS }}=\frac{m_{f}}{\mu} D_{S}^{\text {extS }},
$$

with $\mu=m_{f} m_{C} /\left(m_{f}+m_{C}\right)$. As required, this equation reduces to the extended Smoluchowski model for rigid walls $\left(m_{C}\right.$ $\rightarrow \infty$ ). By modifying the extended Smoluchowski results in this way we can reproduce the simulation results (see Fig. 8). For chain-like molecules the deviation from simulation results is more significant the longer the chain. Maybe the parameters used here are not exactly suitable to represent the more complex molecules. On the other hand for the rigid case the reproduction of the diffusivities is excellent. To get a better understanding of the performance of the different models we use all zero loading results shown in this work and in Ref. 25. Plotting the ratio of the theoretical and simu- lated diffusivities over the TMAC it is shown that the original Smoluchowski model leads to a pronounced overprediction for small $f$ and for $f>0.1$ the deviation is below $50 \%$ [see Fig. 8(c)]. In contrast, the deviation of the modified extended Smoluchowski model is below 10\%, except for pentane and hexane [see Fig. 8(d)]. For this model the best reproduction is obtained for small TMACs.

In this section we showed how to relate simulation results to theoretical results. Modeling all collisions as diffuse (Knudsen model) leads to far too low diffusivities. Modeling the collisions as either specular or diffuse (original Smoluchowski) leads to a pronounced overprediction for very low TMACS. Better results are obtained when a collision is modeled as a combination of a specular and a diffuse collision (extended Smoluchowski). However, the theoretical equation with the fewest parameters and the best performance is the Stokes-Einstein equation (see Ref. 25).

\section{DISCUSSION}

To avoid computationally demanding flexible carbon nanotube simulations we have introduced a method that uses a Lowe-Andersen at interfaces. ${ }^{16,17}$ In this article we have 
extended this methodology to two important classes of problems, namely, mixtures and chain-like molecules. With these extensions the method maintains its simplicity, efficiency, and accuracy. The thermostat reproduces the flexibility influence on diffusion of guest molecules and it gives the correct heat transfer across the interface. To achieve this the parameters have to be chosen carefully. Flexibility influences diffusion in the wall-fluid collision dominated regime, the Knudsen regime. The parameters obtained at the zero loading limit can be used for any other loading. In the fluid-fluid collision dominated regime, the results are not influenced by the thermostat, and the fluid is thermostated at the correct temperature by local thermalization. Here we have to face the fact that we are using the Lowe-Andersen thermostat to change the dynamics of the particles. Of course, we change them so that another assumption underlying these simulations is compensated, namely, the rigid host framework assumption. This is not the usual way thermostats are used, including the Lowe-Andersen thermostat. The LoweAndersen thermostat was originally developed for constant temperature (bulk) dissipative particle dynamics simulations. ${ }^{45}$ Koopman and Lowe ${ }^{48}$ showed how to use it in molecular dynamics simulations in such a way that it does not significantly perturb the dynamics. Here we use it to deliberately perturb the dynamics of adsorbates in such a way as to mimic the effect of thermal motion using an otherwise "frozen" host matrix. In general the main advantages of the Lowe-Andersen thermostat are its simplicity and that it is local. Compared to global thermostats, local thermostats are more realistic and would allow local temperature control.

We also investigated the applicability of theories describing diffusion in the Knudsen regime. By this we relate our simulation results to these theories. In the rarefied gas state the self-diffusivities and transport-diffusivities become equal, and the dynamics are completely described by the self-diffusivities. As the tangential momentum accommodation coefficient is needed for the Smoluchowski and the extended Smoluchowski models, we have introduced a simple concept to calculate this coefficient. It is calculated from standard simulation results, so that no special or extra simulations are needed. The Knudsen diffusivities ${ }^{49}$ are always too low because it is assumed that every collision is diffuse. This theory only holds when the TMAC is close to one. This is not the case for neither flexible CNTs nor rigid CNTs. However, it is valid for very rough pores or when the wallfluid collisions are modeled as diffuse, e.g., with the fluidsolid thermal diffuse scattering algorithm. ${ }^{17,61}$ A better description is given by the Smoluchowski model, ${ }^{50}$ where the collisions are assumed to be either specular or diffuse. The results are still not satisfactory for very low TMACs. Note, as CNTs are relatively smooth, we only investigated systems with low TMACs $(f<0.18)$. A further improvement is provided by the extended Smoluchowski model ${ }^{51}$ which models a collision as partly specular and partly diffuse. It can reproduce results obtained in a rigid CNT. So as to also reproduce the results of the flexible case, we have modified the extended Smoluchowski model. This modification involves a simple term consisting of the fluid mass and the pore-atom mass. Note that the modified version still gives the correct results for the rigid case. Despite the fact that the extended Smoluchowski model gives satisfactory results, the StokesEinstein equation is preferable, in the sense that it gives adequate results ${ }^{25}$ using only one parameter (one which is also used, among others, for the TMAC calculation) and is very simple. By investigating the theoretical approaches, we found an elegant relation between simulations and common theories for the rarefied gas state. By examining these theories, relations between different parameters were clarified. In particular, the TMAC gives some insight as it shows the nature of collisions and gives hints as to the roughness of the interface. The roughness of the host structure has a significant influence on the diffusion of guest molecules. ${ }^{16,51,62}$

Carbon nanotubes are relatively smooth. Consequently, diffusion of molecules inside these materials is rapid. This makes them a promising material for membranes. ${ }^{2}$ Although the diffusion inside carbon nanotubes is much faster than in zeolites (the most used nanoporous material), entrance effects are more important for CNTs than for silicalite. ${ }^{46}$ To realize chemical engineering applications with carbon nanotubes two important questions have to be solved. The toxicity of carbon nanotubes and the handling of it are still not resolved. ${ }^{63}$ Furthermore, one of the most demanding problems to date is CNT synthesis for large scale applications. Nevertheless, as CNT synthesis is a fast-developing field and much research on adsorption and the dynamics of adsorbates is in progress; the field of carbon nanotubes for chemical engineering applications will evolve further in the future.

\section{ACKNOWLEDGMENTS}

This work was supported by the Deutsche Forschungsgemeinschaft (DFG) in priority program SPP 1155 and partially by the EC through the Marie Curie EXT Project MEXT-CT-2005-023311.

${ }^{1}$ J. Kärger and D. M. Ruthven, Diffusion in Zeolites and Other Microporous Solids (Wiley, New York, 1992).

${ }^{2}$ D. S. Sholl and J. K. Johnson, Science 312, 1003 (2006).

${ }^{3}$ B. J. Hinds, N. Chopra, T. Rantell, R. Andrews, V. Gavalas, and L. G. Bachas, Science 303, 62 (2004).

${ }^{4}$ M. J. Casavant, D. A. Walters, J. J. Schmidt, and R. E. Smalley, J. Appl. Phys. 93, 2153 (2003).

${ }^{5}$ A. Srivastava, O. N. Srivastava, S. Talapatra, R. Vajtai, and P. Ajayan, Nat. Mater. 3, 610 (2004).

${ }^{6}$ J. K. Holt, H. G. Park, Y. Wang, M. Stadermann, A. B. Artyukhin, C. P. Grigoropoulos, A. Noy, and O. Bakajin, Science 312, 1034 (2006).

${ }^{7}$ M. Majumder, N. Chopra, R. Andrews, and B. J. Hinds, Nature (London) 438, 44 (2005).

${ }^{8}$ M. S. Dresselhaus, G. Dresselhaus, and P. Avouris, Carbon Nanotubes: Synthesis, Structure, Properties and Applications, Topics in Applied Physics Series Vol. 80 (Springer, Berlin, 2001).

${ }^{9}$ M. Lin, J. P. Y. Tan, C. Boothroyd, K. P. Loh, E. S. Tok, and Y.-L. Foo, Nano Lett. 6, 449 (2006)

${ }^{10}$ W. A. de Heer, P. Poncharal, C. Berger, J. Gezo, Z. Song, J. Bettini, and D. Ugarte, Science 307, 907 (2005).

${ }^{11}$ Y. Wang, M. J. Kim, H. Shan, C. Kittrell, H. Fan, L. M. Ericson, W.-F. Hwang, S. Arepalli, R. H. Hauge, and R. E. Smalley, Nano Lett. 5, 997 (2005).

${ }^{12}$ K. Hata, D. N. Futaba, K. Mizuno, T. Namai, M. Yumura, and S. Iijima, Science 306, 1362 (2004).

${ }^{13}$ B. Wei, R. Vajtai, Y. Jung, J. Ward, R. Zhang, G. Ramanath, and P. M. Ajayan, Nature (London) 416, 495 (2002).

${ }^{14}$ D. Frenkel and B. Smit, Understanding Molecular Simulations, From Algorithms to Applications (Academic, San Diego, 2002).

${ }^{15}$ M. P. Allen and D. J. Tildesley, Computer Simulation of Liquids (Clar- 
endon, Oxford, 1987)

${ }^{16}$ S. Jakobtorweihen, M. G. Verbeek, C. P. Lowe, F. J. Keil, and B. Smit, Phys. Rev. Lett. 95, 044501 (2005).

${ }^{17}$ S. Jakobtorweihen, C. P. Lowe, F. J. Keil, and B. Smit, J. Chem. Phys. 124, 154706 (2006).

${ }^{18}$ S. Andreev, D. Reichman, and G. Hummer, J. Chem. Phys. 123, 194502 (2005).

${ }^{19}$ A. Kalra, S. Garde, and G. Hummer, Proc. Natl. Acad. Sci. U.S.A. 100, 10175 (2003).

${ }^{20}$ C. Wei and D. Srivastava, Phys. Rev. Lett. 91, 235901 (2003).

${ }^{21}$ V. P. Sokhan, D. Nicholson, and N. Quirke, J. Chem. Phys. 120, 3855 (2004).

${ }^{22}$ A. I. Skoulidas, D. M. Ackerman, J. K. Johnson, and D. S. Sholl, Phys. Rev. Lett. 89, 185901 (2002)

${ }^{23}$ H. Chen, J. K. Johnson, and D. S. Sholl, J. Phys. Chem. B 110, 1971 (2006).

${ }^{24}$ H. Chen and D. S. Sholl, J. Am. Chem. Soc. 126, 7778 (2004).

${ }^{25}$ S. Jakobtorweihen, F. J. Keil, and B. Smit, J. Phys. Chem. B 110, 16332 (2006).

${ }^{26}$ K.-H. Lee and S. B. Sinnott, Nano Lett. 5, 793 (2005).

${ }^{27}$ K.-H. Lee and S. B. Sinnott, J. Phys. Chem. B 108, 9861 (2004).

${ }^{28}$ T. Düren, F. J. Keil, and N. A. Seaton, Mol. Phys. 100, 3741 (2002).

${ }^{29}$ M. W. Roth and J. Mesentseva, Mol. Simul. 30, 661 (2004).

${ }^{30}$ D. Cao and J. Wu, Langmuir 20, 3759 (2004).

${ }^{31}$ G. Arora and S. I. Sandler, J. Chem. Phys. 124, 084702 (2006).

${ }^{32}$ G. Arora, N. J. Wagner, and S. I. Sandler, Langmuir 20, 6268 (2004).

${ }^{33}$ R. E. Tuzun, D. W. Noid, B. G. Sumpter, and R. C. Merkle, Nanotechnology 7, 241 (1996).

${ }^{34}$ R. Krishna and J. M. van Baten, Ind. Eng. Chem. Res. 45, 2084 (2006)

${ }^{35}$ S. Supple and N. Quirke, Phys. Rev. Lett. 90, 214501 (2003).

${ }^{36}$ H. Yang, Y. Liu, H. Zhang, and Z.-S. Li, Polymer 47, 7607 (2006).

${ }^{37}$ S. B. Sinnott, Z. Mao, and K.-H. Lee, Comput. Model. Eng. Sci. 3, 575 (2002).

${ }^{38}$ Z. Mao and S. B. Sinnott, J. Phys. Chem. B 105, 6916 (2001).

${ }^{39}$ Z. Mao and S. B. Sinnott, J. Phys. Chem. B 104, 4618 (2000).

${ }^{40}$ F. Zhang, J. Chem. Phys. 111, 9082 (1999).

${ }^{41}$ S. Y. Bhide and S. Yashonath, J. Am. Chem. Soc. 125, 7425 (2003).
${ }^{42}$ J. Jiang, S. I. Sandler, M. Schenk, and B. Smit, Phys. Rev. B 72, 045447 (2005).

${ }^{43}$ J. Jiang, S. I. Sandler, and B. Smit, Nano Lett. 4, 241 (2004).

${ }^{44}$ A. Heyden, T. Düren, and F. J. Keil, Chem. Eng. Sci. 57, 2439 (2002).

${ }^{45}$ C. P. Lowe, Europhys. Lett. 47, 145 (1999).

${ }^{46}$ D. A. Newsome and D. S. Sholl, Nano Lett. 6, 2150 (2006).

${ }^{47}$ C. P. Lowe and M. W. Dreischor, in Novel Methods in Soft Matter Simulations, Lecture Notes in Physics, Vol. 640 edited by M. Karttunen, I. Vattulainen, and A. Lukkarinen (Springer, Berlin, 2004), pp. 39-68.

${ }^{48}$ E. A. Koopman and C. P. Lowe, J. Chem. Phys. 124, 204103 (2006).

${ }^{49}$ M. Knudsen, Ann. Phys. 28, 75 (1909).

${ }^{50}$ M. v. Smoluchowski, Ann. Phys. 33, 1559 (1910).

${ }^{51}$ G. Arya, H.-C. Chang, and E. J. Maginn, Phys. Rev. Lett. 91, 026102 (2003).

${ }^{52}$ R. Saito, M. Fujita, G. Dresselhaus, and M. S. Dresselhaus, Appl. Phys. Lett. 60, 2204 (1992).

${ }^{53}$ J. H. Walther, R. Jaffe, T. Halicioglu, and P. Koumoutsakos, J. Phys. Chem. B 105, 9980 (2001)

${ }^{54}$ M. G. Martin and J. I. Siepmann, J. Phys. Chem. B 102, 2569 (1998).

${ }^{55}$ S. K. Nath, F. A. Escobedo, and J. de Pablo, J. Chem. Phys. 108, 9905 (1998).

${ }^{56}$ W. A. Steele, The Interaction of Gases with Solid Surfaces (Pergamon, Oxford, 1974).

${ }^{57}$ M. E. Tuckerman, B. J. Berne, and G. J. Martyna, J. Chem. Phys. 97, 1990 (1992)

${ }^{58}$ G. J. Martyna, M. E. Tuckerman, D. J. Tobias, and M. L. Klein, Mol. Phys. 87, 1117 (1996).

${ }^{59}$ D. Dubbeldam, S. Calero, T. Maesen, and B. Smit, Phys. Rev. Lett. 90, 245901 (2003).

${ }^{60}$ G. Arya, H.-C. Chang, and E. J. Maginn, Mol. Simul. 19, 697 (2003).

${ }^{61}$ J. M. D. MacElroy and M. J. Boyle, Chem. Eng. J. 74, 85 (1999).

${ }^{62}$ K. Malek and M.-O. Coppens, Phys. Rev. Lett. 87, 125505 (2001).

${ }^{63}$ C.-W. Lam, J. T. James, R. McCluskey, S. Arepalli, and R. L. Hunter, Crit. Rev. Toxicol. 36, 189 (2006).

${ }^{64}$ B. E. Poling, J. M. Prausnitz, and J. P. O'Connell, The Properties of Gases and Liquids, 5th ed. (McGraw-Hill, New York, 2000). 